\title{
Mid-infrared coincidence measurements based on intracavity frequency conversion
}

Piccione, S.; Mancinelli, M.; Trenti, A.; Fontana, G.; Dam, J.; Tidemand-Lichtenberg, Peter; Pedersen, C.; Pavesi, L.

Published in:

Nonlinear Frequency Generation and Conversion: Materials and Devices XVII

Link to article, DOI:

$10.1117 / 12.2305713$

Publication date:

2018

Document Version

Peer reviewed version

Link back to DTU Orbit

Citation (APA):

Piccione, S., Mancinelli, M., Trenti, A., Fontana, G., Dam, J., Tidemand-Lichtenberg, P., Pedersen, C., \& Pavesi, L. (2018). Mid-infrared coincidence measurements based on intracavity frequency conversion. In K. L. V., \& K. L. S. (Eds.), Nonlinear Frequency Generation and Conversion: Materials and Devices XVII SPIE - International Society for Optical Engineering. Proceedings of SPIE - The International Society for Optical Engineering https://doi.org/10.1117/12.2305713

\section{General rights}

Copyright and moral rights for the publications made accessible in the public portal are retained by the authors and/or other copyright owners and it is a condition of accessing publications that users recognise and abide by the legal requirements associated with these rights.

- Users may download and print one copy of any publication from the public portal for the purpose of private study or research.

- You may not further distribute the material or use it for any profit-making activity or commercial gain

- You may freely distribute the URL identifying the publication in the public portal 


\title{
Mid-infrared coincidence measurements based on intracavity frequency conversion
}

\author{
S.Piccione ${ }^{\mathrm{a}}$, M.Mancinelli ${ }^{\mathrm{a}, \mathrm{c}}$, A.Trenti ${ }^{\mathrm{a}}$, G.Fontana ${ }^{\mathrm{a}}$, J.Damb ${ }^{\mathrm{b}}$, P. Tidemand-Lichtenbergb ${ }^{\mathrm{b}}$, C. \\ Pedersen $^{\mathrm{b}}$, and L. Pavesi ${ }^{\mathrm{a}}$

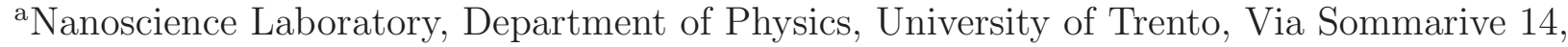 \\ Trento 38123, Italy \\ ${ }^{\mathrm{b}}$ DTU Fotonik, Department of Photonics Engineering, Technical University of Denmark, \\ Roskilde 4000, Denmark \\ ${ }^{c}$ Research Programs, SM Optics s.r.l., via John Fitzgerald Kennedy 2, Vimercate 20871, Italy
}

\begin{abstract}
In the last years, the Mid Infrared (MIR) spectral region has attracted the attention of many areas of science and technology, opening the way to important applications, such as molecular imaging, remote sensing, freespace communication and environmental monitoring. However, the development of new sources of light, such as quantum cascade laser, was not followed by an adequate improvement in the MIR detection system, able to exceed the current challenges. Here we demonstrate the single-photon counting capability of a new detection system, based on efficient up-converter modules, by proving the correlated nature of twin photons pairs at about $3.1 \mu \mathrm{m}$, opening the way to the extension of quantum optics experiments in the MIR.
\end{abstract}

Keywords: MIR, coincidence measurements, frequency conversion, nonlinear optics, spontaneous parametric down conversion,MIR single photon detector,room temperature MIR detector, MIR quantum optics

\section{INTRODUCTION}

The MIR spectral region, which ranges from $2 \mu \mathrm{m}$ to $20 \mu \mathrm{m}$, is becoming a technologically important spectral band since it covers both the atmospheric windows $(3-5 \mu \mathrm{m}$ and $8-12 \mu \mathrm{m})$, relevant for free space communication [1] and environmental monitoring [2] and encompasses the primary absorption bands of nearly all gas molecule, of great interest for sensoristic and spectroscopy application. Significant improvements can be expected with the use of MIR spectroscopy, such as the possibility of on-line detection of numerous different molecules creating a new powerful diagnostic tool [3]. Moreover, the spectral radiance of room temperature objects falls into the MIR region, promoting the realization of novel thermal camera application [4]. Also in integrated photonics a lot of efforts has been done to extend the operational wavelength of the silicon platform [5], which could revolutionize the MIR-optics and offers a great growth opportunity for integrated photonics [6]. Thus, the unique and attractive characteristic of the MIR range call for enabling techniques to yield sensitive detection. While already available for their visible and near infrared (NIR) counterpart, where manipulation and detection of photons down to the level of single-photon measurements are a mature technology, MIR optical detection systems suffer from considerably inferior performance. The main limit is caused by the inherent sensitivity to the unwanted incident black body radiation coming from the environmental space and dark current, induced by the finite temperature of the detector itself. A solution is offered when the detector is cooled down, reducing these two noise sources. However the results is an expensive and bulky device. Additionally, even when cooled, the MIR detectors show poor signal-to-noise ratio (SNR), if compared to NIR and visible light detectors [7]. A way to increase the sensitivity involve the use of superconducting nanowire single-photon detectors (SNSPDs) [8], with a reduced width. However the system detection efficiency drops and the dark current increases [9]. All these considerations lead to the absence of a MIR detector, capable of performing a single-photon level measurement.

Further author information: (Send correspondence to S.P)

S.P: E-mail: sara.piccione@unitn.it 
An example of the importance of single-photon counting measurements can be seen in the realization of experiments in quantum optics with unprecedented precision [10]. One of the key experiments to determine the quantum properties of light involves the coincidence detection of photon pairs. Extending the quantum optics also in the MIR, could lead to new applications for quantum sensing, quantum communication and quantum imaging.

For this reason it is now urgent the presence of a MIR single-photon detector.

The solution we propose involves the concept of spectral translation. The idea is to up-convert the infrared radiation into visible radiation by means of optical nonlinear effects[11]. The incoming signal is then shifted to a wavelength interval where efficient and low noise single photon detectors are already present, so that the unwanted influence of the environmental radiation is reduced. This enabled us to demonstrate coincidence measurements on correlated photon pairs at $3.1 \mu \mathrm{m}$.

\section{THE DETECTOR}

The nonlinear process which governs the operation of the module is the $\chi^{(2)}$ nonlinear process of sum frequency generation. Two optical waves are mixed into a Periodically Poled Lithium Niobate (PPLN) nonlinear crystal, $20 \mathrm{~mm}$ long, in order to generate a third wave whose frequency is the sum of the two. In a periodically poled material the orientation of one of the crystalline axis is periodically inverted as a function of the position. This causes an inversion of the sign of the nonlinear coefficient, which is able to compensate the wavevector mismatch of all the waves involved in the process. This technique is known as Quasi-Phase-Matching and allows the reaching of high conversion efficiency [12].

The crystal was fabricated from Covesion Ltd. for sum frequency generation between a $1.064 \mu \mathrm{m}$ pump laser beam and an incident radiation in the $3 \mu \mathrm{m}$ range. It contains five poled regions whose periods range from 21 to $23 \mu \mathrm{m}$ in steps of $0.5 \mu \mathrm{m}$, with a size of $1 \mathrm{~mm} \times 1 \mathrm{~mm}$. To reduce reflection losses, the crystal is AR-coated for both the pump and the MIR beam. The fine tuning of the phase-match condition is achieved thanks to a temperature controller. Figure 1 shows the expected dependence of the up-conversion efficiency as a function of the crystal temperature, for each of the poled region. From 1 it appears another feature that characterize the

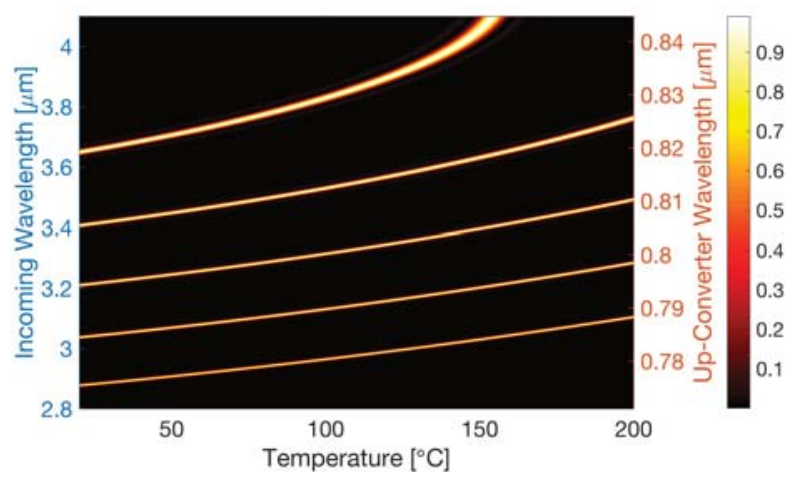

Figure 1. Efficiency as a function of both the phase matched wavelength and the crystal temperature, for the different poling periods.

system: its tunability to any wavelength within the transparency range of the nonlinear crystal.

The efficiency of the process, defined as the ratio between the intensity of up-converter radiation and the intensity of the incoming signal, depends on the intensity of the pump beam. The idea of up-conversion for infrared imagining is not new [13], but because of the requirements of a high power pump laser source, its use for practical applications was thought not to be possible, due to the extremely low conversion efficiency. Things have changed thanks to a recent achievement demonstrating a measured quantum efficiency of $20 \%$, almost six orders of magnitude higher than the values previously reported [14]. The revolutionary idea was to place the nonlinear crystal inside a laser cavity, which guarantees a high circulating power and low losses. The up-converter module comprises different elements (see Figure 2). The up-conversion crystal (1 in Figure 2) is placed inside a high 


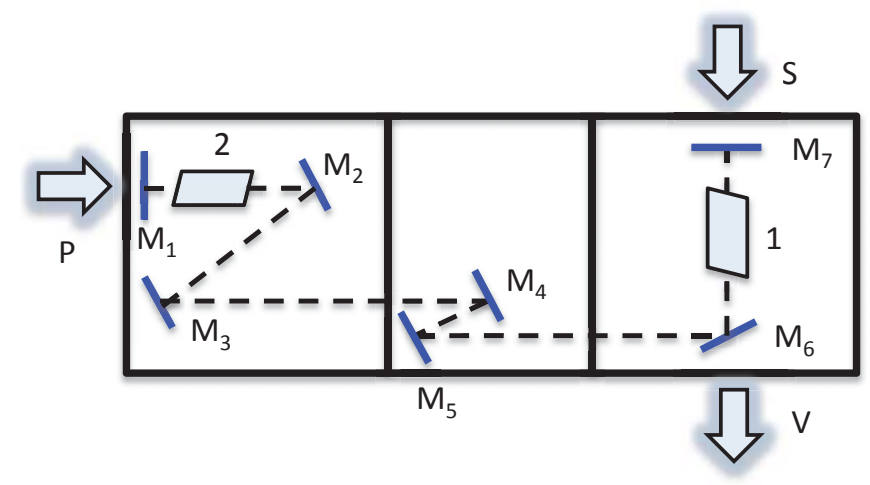

Figure 2. Internal view of the up-conversion module. M1-M7 indicates the cavity mirrors, 1 stands for the PPLN crystal, while 2 indicates the $N d: Y V O_{4}$ crystal. $\mathrm{P}$ indicates the pump beam, $\mathrm{S}$ the incoming MIR signal and $\mathrm{V}$ the up-converted visible light.

finesse folded fabry-perot $1.064 \mu \mathrm{m}$ laser cavity so to enhance the quantum efficiency. The laser cavity consists of a $N d: \mathrm{YVO}_{4}$ crystal (2 in Figure 2) pumped by a $4 \mathrm{~W}$ broad area diode laser at $880 \mathrm{~nm}$. The cavity includes seven mirrors (M1-M7 in Figure 2), to be configurable for different spot size and to remove residual pump pho-

tons. With this configuration it is possible to achieve up to $100 \mathrm{~W}$ of circulating power. The configurable spot dimension is of great importance since the efficiency of the nonlinear process depends on the intensity of all the beams involved. Moreover, the efficiency is higher when the waists of both the pump and the signal are equal. Moreover the phase-matched wavelength is also function of the relative angle between the incoming MIR signal and the pump beam. Therefore, the optical alignment becomes a crucial parameter, influencing the performance of the novel detector.

Finally, the generated visible light exits from the up-converter module through the mirror $M_{6}$ and could be detected with a visible photon detector.

The MIR single-photon counter is, then, realized with the combination of the up-converter module, described above, with a state-of-the-art silicon single photon avalanche photodiode(SPAD). The quantum efficiency (QE) of the detector is given by the product between the measured conversion efficiency (10\%) and the SPAD QE (65\%, while the time resolution depends only on the electronics of the SPAD and not by the conversion process, which can be considered to happen instantaneously. Moreover the detector does not require the cryo-cooling to operate properly.

One of the major characteristics the up-conversion process is the fact that the process is characterized by a narrow bandwidth $(\sim 20 \mathrm{~nm})$. For this reason the frequency conversion inherently acts both as a spectral and spatial filter to the incoming MIR radiation. This particular feature is in strong contrast to direct MIR detection system and allows both spatial and spectral resolved measurements at the single-photon level.

On the other hand, the fact that a detector has a limited bandwidth might also have some drawbacks. If the incoming signal is a broad-band MIR source, it is not possible to get the entire power in a single measurement and the signal reconstruction through the scan of the phase matching condition is necessary.

\section{EXPERIMENTAL SET-UP}

To demonstrate the capability of the detection unit, we measure the time correlation of MIR photon pairs by means of a coincidence measurement. Coincidence counting generally involves two or more detectors connected to an electronic coincidence circuit. When a detector measures a photon, it triggers a signal in the form of an electrical pulse. The temporal coincidence between signals from two separate photon detectors will reveal if there is a correlation in the arrival time of the photons at the detectors.

The twin photons are generated in a free space set-up through the second order nonlinear process of Spontaneous Parametric Down Conversion (SPDC). In the SPDC process a pump photon, when traveling through a nonlinear material, is spontaneously converted into two photons of lower energy, historically named signal and idler. The 
essential feature of the process is that the two generated photons are correlated in momentum, energy and time. Depending on the frequency of the two generated photons we can distinguish between the non-degenerate process, in which the two are different, and the degenerate process, where signal and idler have the same wavelength. Besides the frequency, another difference between the two process regards the different bandwidth of the two, equal to $50 \mathrm{~nm}$ for the non degenerate case and $200 \mathrm{~nm}$ for the degenerate process. The nonlinear material is again a PPLN crystal, $10 \mathrm{~mm}$ long and containing sixteen periodically poled regions ranging from 24.06 to $36.95 \mu \mathrm{m}$, each with a $500 \times 500 \mu \mathrm{m}$ aperture. The occurrence of the degenerate process instead of the non degenerate one depends on the crystal temperature. Figure 3 shows the efficiency of the SPDC process as a function of both the temperature of the nonlinear crystal and the generated wavelength, for a poled region of period $L_{p}=34.68 \mu \mathrm{m}$.

From a comparison with the bandwidth of the up-conversion module, it is clear that the detection system is

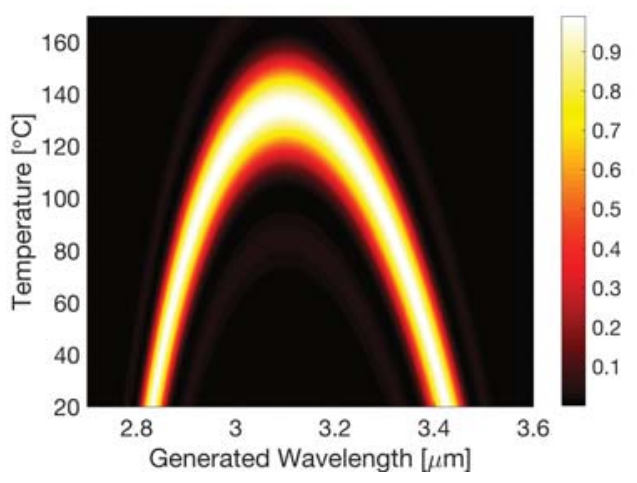

Figure 3. Simulated bandwidth as a function of temperature and wavelength for $L_{p}=34.68 \mu \mathrm{m}$.

more efficient for the non-degenerate process, since the process bandwidth is close to that of up-conversion.

The experimental set-up, figured in Figure 4 used for the coincidence measurement consists of a fiber-coupled, continuous-wave laser with tuning range from 1.520 to $1.565 \mu \mathrm{m}$ and maximum output power of $70 \mathrm{~mW}$.

The laser source is amplified using an erbium-doped fiber amplifier with maximum output power of $800 \mathrm{~mW}$.

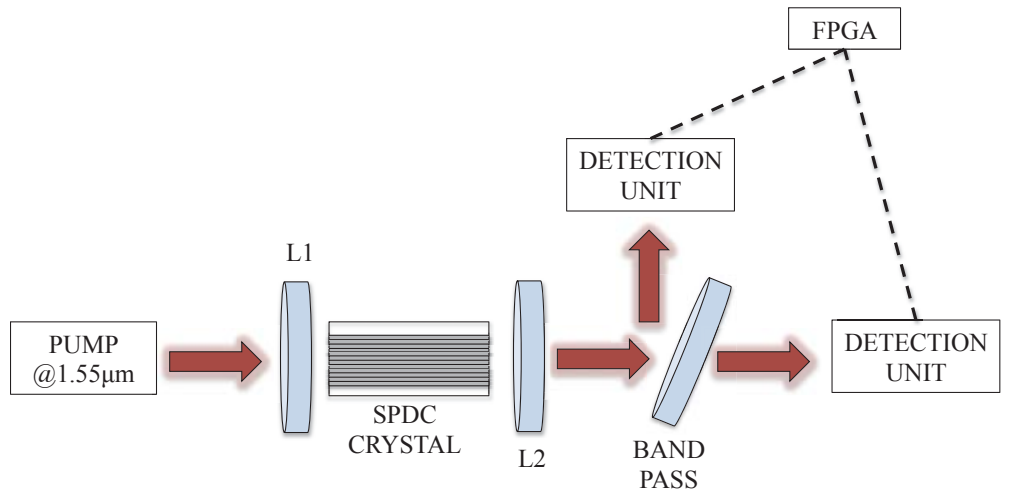

Figure 4. Sketch of the experimental set-up. A continuous-wave infrared laser pumps a PPLN crystal.Lens L1 has a focal length of $150 \mathrm{~mm}$ and focuses the beam, whose waist before L1 is $2.25 \mathrm{~mm}$, to a beam waist of $35 \mu \mathrm{m}$ in the PPLN crystal. The PPLN crystal contains different poled regions each with a transverse cross section of $500 \mathrm{~mm}$ by $500 \mathrm{~mm}$. The nominal poling period used is $34.48 \mu \mathrm{m}$. The generated MIR photon pairs and the residual $1.55 \mathrm{~mm}$ pump are collimated by a $\mathrm{CaF}_{2}$ converging lens L2, whose focal length is $18 \mathrm{~mm}$. The generated photon-pairs are split by an optical band-pass filter centered at $3 \mu \mathrm{m}$, with a bandwidth of $500 \mathrm{~nm}$. The idler wavelength at $3.343 \mu \mathrm{m}$ is reflected and sent to one up-conversion detection module, while the signal photon at $2.89 \mu \mathrm{m}$ is transmitted through the band-pass filter to the second up-conversion detection module.

We controlled the polarization of the light from the laser with a free-space polarization controller stage composed 
by two quarter-wave plates and one half-wave plate. The pump beam at $1.55 \mathrm{~mm}$ is focused by a first lens ( $\mathrm{f}=15$ $\mathrm{cm}$ ) into PPLN, crystal pumping the SPDC process. It was proved experimentally that the optimum condition is achieved when the Rayleigh range is approximately half the length of the crystal. The temperature of the nonlinear crystal is controlled with $0.1^{\circ} \mathrm{C}$ accuracy from just above room temperature to $200^{\circ} \mathrm{C}$. The oven housing with the nonlinear crystal can be translated, allowing for selection of the required poling period. The generated photon-pairs are then split and directed to the two modules, using an IR optical band-pass filter centered at 3 $\mathrm{mm}$, with a bandwidth of $500 \mathrm{~nm}$. The idler wavelength at $3.343 \mu \mathrm{m}$ is reflected and the signal photon at $2.89 \mu \mathrm{m}$ is transmitted through the band-pass filter. Residual pump photons are eliminated by a Germanium window, placed at the entrance of each of the up-conversion modules. To maximize the efficiency of the up-conversion, it is necessary for the incoming MIR beam to have a beam waist comparable to the beam waist of the laser beam in the up-conversion module. This is done thanks to a lens of focal length $f=2 \mathrm{~cm}$. The up-converted photons are detected using silicon-based, single-photon counters (SPCM-AQRH Excelitas), each coupled to an up-converter module. The SPCM-AQRH is based on a silicon avalanche photodiode with a circular active area, achieving a peak photon detection efficiency of about $65 \%$ at $792 \mathrm{~nm}$ over a $180 \mathrm{~mm}$ diameter with unmatched uniformity over the full active area.

The output TTL voltage of the two SPADs is fed into a Field Programmable Gate Array (FPGA) digital correlator that provides the coincidence rate. The FPGA is programmed to make a real-time cross-correlation between the TTL signals of the two Si SPADs.

\section{RESULTS}

Figure 5 shows the signal collected by a single detection unit. The crystal temperature of the up-conversion module was set equal to $T=145^{\circ}$ in order to phase match an icoming radiation of wavelength equal to $\lambda=3.1 \mu \mathrm{m}$. It was estimated an incoming power of $P=5.8 \pm 0.1 \mathrm{pW}$.

As demonstrated in [15] a clear peak of coincidence was observed. The coincidence window was equal to $1.33 \mathrm{~ns}$

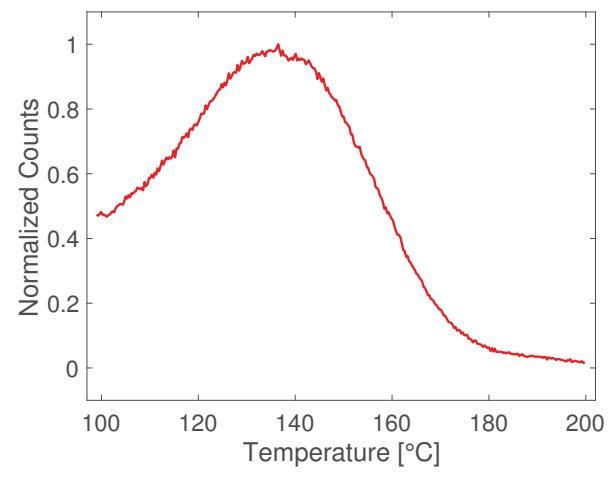

Figure 5. Collected signal from a single detection unit[15].

and a peak of rate of $105 \pm 1$ cps was measured, after $10 \mathrm{~s}$ of integration.

The signal-to-noise ratio in coincidence measurements is usually named coincidence to accidental ratio (CAR). CAR is calculated as the number of coincidences within the coincidence window divided by the average of the background counts on the same time window taken apart from the peak [16]. A CAR of $15.7 \pm 0.4 c p s$ and an accidental mean rate of coincidence of $6.7 \pm 0.1$ cps.

The measure was repeated for different values of pump power. There is a trade-off in the maximum pump power that can be used for the SPDC process to maximize the CAR. In the low pump power regime, the number of generated photon pairs through the SPDC process increases linearly with the pump power [17]. However, above a certain threshold the generation of more than one pair within the same time-slot will decrease the CAR. This process is called multipair emission in SPDC and it is well known to have a detrimental effect on the coincidence measurement [18].

The experimental coincidence measurement was performed with an incident pump power set to an optimal value 
of $70 \mathrm{~mW}$ [15]. The conversion efficiency of the SPDC process is estimated to be $\eta=-110 \mathrm{~dB}$. After optimization of the temperatures of the PPLN crystals, the count rate for both modules is respectively $153 k c p s$ and $150 k c p s$, with a background of $9 k c p s$ and $14 k c p s$. The main contribution to the background count rate in both modules originates from spurious signals at the output of the module itself.

We estimated a total loss of $25 \mathrm{~dB}$ from the output facet of the SPDC crystal to the input of the single-photon detectors. Approximately $10 \mathrm{~dB}$ originates from the conversion efficiency of both modules, which is $10 \%$. This value can be increased up to $20 \%$ by a suitable optimization of the module [14]. One of the used lens is made of $\mathrm{CaF}_{2}$ material, which is transparent at the relevant mid-IR wavelengths and accounts for a loss of $0.45 \mathrm{~dB}$. The filters used after the module account for $0.5 \mathrm{~dB}$. They are short pass (cut-off at 1000nm), short pass (cut-off at $850 \mathrm{~nm}$ ), long pass (cut-off at $600 \mathrm{~nm}$ ) and long pass (cut-off at $750 \mathrm{~nm}$ ) wavelength filters. The background count rate of each module is mainly due to spurious signals coming from the module itself.

The detection efficiency of the silicon single photon counter is $65 \%(1.9 d B)$. The remaining $12.15 d B$ losses are due to a non perfect overlap with the active area of the silicon detector, which has $180 \mu m$ diameter. In our implementation, a tighter focus was not feasible, due to intrinsic limiting lens aberrations, amplified at shorter focal lengths.

\section{FIBER COUPLING}

The compactness of the system can be further increased by coupling both the input and the output of the upconverter module to optical fibers. This will considerably simplify the measurements as well as will drastically reduce the coupling losses to the SPADs, which represents the major contributions to the total losses of the system.

Since a MIR laser was not available in the lab, we decided to use the up-converter module itself as the pump source. In fact, the phase-matching conditions are the same for both the nonlinear processes of sum-frequency generation and difference-frequency generation (DFG). DFG is a second order nonlinear process, in which optical waves at frequency $\omega_{1}$ and $\omega_{2}$ interacts inside the nonlinear medium, to generate a third wave whose frequency is given by $\omega_{3}=\omega_{2}-\omega_{1}$. Therefore, by using DFG a tunable MIR laser source can be realized combining a tunable visible $T i: S a(\lambda=700 \div 1000 \mathrm{~nm})$ laser and the module. Figure 6 shows the converted MIR power as a function of the incident visible light, at a wavelength of $3.1 \mu \mathrm{m}$. Once the source was realized, we have to design carefully

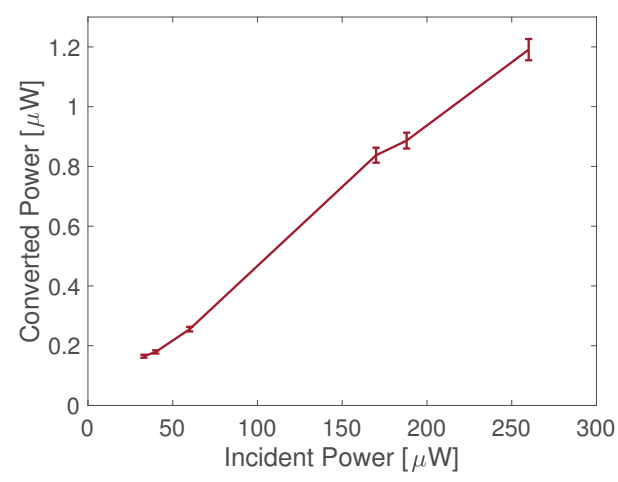

Figure 6. MIR Converted Power as a function of the VIS incident power.

the system since we have to perfectly overlap the beam traveling from the input fiber towards the PPLN crystal in the module and the pump beam of the module itself. To achieve a high conversion efficiency, necessary for single photon counting operation, the two beams should have the same dimension. The beam radius of the pump beam is equal to $180 \mu \mathrm{m}$, while the radius of the beam exiting from the collimator is equal to $1.2 \mathrm{~mm}$. To make the two comparable in the nonlinear crystal a particular focusing and positioning system was design so to keep the detection unit compact. The design is currently under testing. 


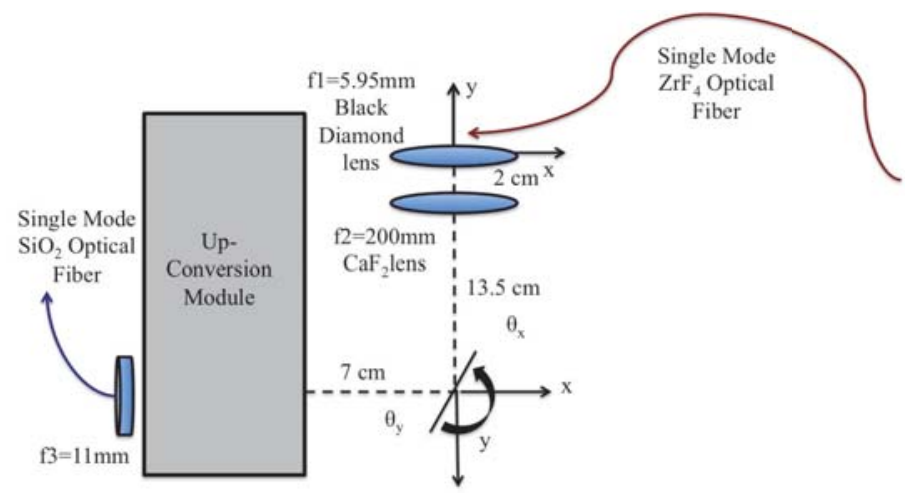

Figure 7. Sketch of the set-up for iber coupling. $f_{1}, f_{2}$ and $f_{3}$ indicates the focal length of the used lenses.

\section{CONCLUSION}

We have presented a room temperature coincidence measurement of correlated MIR photons. Our detection system outperforms single-photon detectors based on superconducting nanowires in the MIR, the only technology sensitive enough to allow single photon counting operations, in terms of quantum efficiency, speed and noise. This is a key result because coincidence measurement is the standard technique today for photon pair detection, which is at the core of many quantum optics experiments, and it is essential in quantum communication protocols based on entangled photons [19]. The proposed system is also a part of a proof of concept towards fully integrated devices. In fact, the integration of an up-converter module on a silicon chip is possible by using silicon SPADs coupled to an up-converter stage realized in the $\mathrm{SiN}$ or $\mathrm{SiON}$ alloy based on third order nonlinear effects. The results reported here paves the way to quantum optical applications in the MIR, where the ability to perform coincidence measurement is a cornerstone.

\section{REFERENCES}

[1] Bellei, F., Cartwright, A. P., McCaughan, A. N., Dane, A. E., Najafi, F., Zhao, Q., and Berggren, K. K., "Free-space-coupled superconducting nanowire single-photon detectors for infrared optical communications," Optics express 24(4), 3248-3257 (2016).

[2] Weibring, P., Edner, H., and Svanberg, S., "Versatile mobile lidar system for environmental monitoring," Appl. Opt. 42, 3583-3594 (Jun 2003).

[3] Fernandez, D. C., Bhargava, R., Hewitt, S. M., and Levin, I. W., "Infrared spectroscopic imaging for histopathologic recognition," Nature biotechnology 23(4), 469-474 (2005).

[4] Daffara, C., Ambrosini, D., Pezzati, L., and Paoletti, D., "Thermal quasi-reflectography: a new imaging tool in art conservation," Opt. Express 20, 14746-14753 (Jun 2012).

[5] Soref, R., "Mid-infrared photonics in silicon and germanium," Nature photonics 4(8), 495-497 (2010).

[6] Castellan, C., Trenti, A., Mancinelli, M., Marchesini, A., Ghulinyan, M., Pucker, G., and Pavesi, L., "From shg to mid-infrared spdc generation in strained silicon waveguides," in [Quantum Photonic Devices], 10358, 1035804, International Society for Optics and Photonics (2017).

[7] Rogalski, A., "Infrared detectors: status and trends," Progress in quantum electronics 27(2), 59-210 (2003).

[8] Marsili, F., Verma, V. B., Stern, J. A., Harrington, S., Lita, A. E., Gerrits, T., Vayshenker, I., Baek, B., Shaw, M. D., Mirin, R. P., et al., "Detecting single infrared photons with $93 \%$ system efficiency," Nature Photonics 7(3), 210-214 (2013).

[9] Marsili, F., Bellei, F., Najafi, F., Dane, A. E., Dauler, E. A., Molnar, R. J., and Berggren, K. K., "Efficient single photon detection from $500 \mathrm{~nm}$ to $5 \mu \mathrm{m}$ wavelength," Nano letters 12(9), 4799-4804 (2012).

[10] Hadfield, R. H., "Single-photon detectors for optical quantum information applications," Nature photonics 3(12), 696-705 (2009).

[11] Midwinter, J., "Image conversion from $1.6 \mu$ to the visible in lithium niobate," Applied Physics Letters 12(3), 68-70 (1968). 
[12] Fejer, M. M., Magel, G., Jundt, D. H., and Byer, R. L., "Quasi-phase-matched second harmonic generation: tuning and tolerances," IEEE Journal of Quantum Electronics 28(11), 2631-2654 (1992).

[13] Lucy, R., "Infrared to visible parametric upconversion," Applied optics 11(6), 1329-1336 (1972).

[14] Dam, J. S., Tidemand-Lichtenberg, P., and Pedersen, C., "Room-temperature mid-infrared single-photon spectral imaging," Nature photonics 6(11), 788-793 (2012).

[15] Mancinelli, M., Trenti, A., Piccione, S., Fontana, G., Dam, J., Tidemand-Lichtenberg, P., Pedersen, C., and Pavesi, L., "Mid-infrared coincidence measurements on twin photons at room temperature," Nature Communications 8 (2017).

[16] Davanco, M., Ong, J. R., Shehata, A. B., Tosi, A., Agha, I., Assefa, S., Xia, F., Green, W. M., Mookherjea, S., and Srinivasan, K., "Telecommunications-band heralded single photons from a silicon nanophotonic chip," Applied Physics Letters 100(26), 261104 (2012).

[17] Suhara, T. and Kintaka, H., "Quantum theory analysis of twin-photon beams generated by parametric fluorescence," IEEE journal of quantum electronics 41(9), 1203-1212 (2005).

[18] Broome, M. A., Almeida, M. P., Fedrizzi, A., and White, A. G., "Reducing multi-photon rates in pulsed down-conversion by temporal multiplexing," Optics express 19(23), 22698-22708 (2011).

[19] Jennewein, T., Simon, C., Weihs, G., Weinfurter, H., and Zeilinger, A., "Quantum cryptography with entangled photons," Physical Review Letters 84(20), 4729 (2000). 